have evaluated the association between the individual MetS components and the odds of PCa diagnosis, clinically significant PCa, and high-grade PCa in a cohort of 2235 Canadian patients referred for biopsy. No individual MetS component was independently associated with PCa. However, increasing number of Mets components was associated with higher probability of PCa, clinically significant PCa and high grade PCa diagnosis. Although this is a retrospective study and the information on patients' hormonal milieu was not available, it improves our knowledge on the association of MetS and PCa by suggesting that there is a continuum of risk with MetS rather than an "all-or-none" phenomenon. The question whether the presence of MetS should change current PCa assessment and biopsy indications is yet to be found.

\title{
Re: Social Media and Online Communication: Clinical Urology Practice in the 21st Century
}

\section{Michael J. Ehlert}

Urology Parctice 2015 Jan;2: 2-6.

\section{EDITORIAL COMMENT}

The world has witnessed an explosion of social media in recent years, in both personal and professional terms. Social media use among urologists also remains high with more than 66\% reporting to have an active account. In this review Michael J Ehlert describes the available social media types such as Twitter, Facebook, YouTube, Pinterest as well as Blogs and personal websites, provides information on how and why to use these platforms, and insight to the risks and challenges that may be faced by medical personnel who use the social media.

\section{Re: Real-Time Elastography of the Prostate}

\section{Junker, T.D. Zordo, M. Quentin, M. Ladurner et al.}

Bio Med Research International vol 2014, Article ID 180804, 11 pages.

\section{EDITORIAL COMMENT}

Elastography was described in 1991 by Ophir. Palpation of tissue is the oldest physical examination as palpation of the breast or prostate (DRE). Hard palpable tissues are suspicious for cancer and this is basic principle of the real-time elastography (RTE). It is a ultrasound technique and is able to visualise tissue elasticity. Despite of low spesificity of PSA and low sensitivity of systematic biopsy, they are gold standarts for PCa diagnosis. Nowadays, PCa can detected with high sensitivity by multiparametric MRI or contrast-enhanced TRUS. On the other hand, real time elastography can be prefered because of chep and non invasive. Strainelastography (red; softtissue, blue; hard tissue) and shear-waveelastography (35-37 kPA; kilopascal) were described. Prostatitis, fibrozis, BPH may be associated with increased tissue stiffness can be responsible for low positive predictive values as 39\%. Some authors reported best performance of the SWE elastography as sensitivity (63\%), specificity (91\%), PPV (69.4\%) and NPV (91\%). RTE targeted biopsy can be recommended for detection of PCa but nowadays it is not standard. RTE may be particularly suitable for detection of clinically significant cancers. RTE may misss carcinomas with a primary Gleason pattern of 3 (falsenegative). In the future, elastography techniques will developed by moresensitive probes and it can be helpful for detection of PCa additional to DRE. 